Historic, Archive Document

Do not assume content reflects current scientific knowledge, policies, or practices. 


\section{WERTER DEVAUGHN}

\section{Seed Grower, Importer and Merchant}

\section{Office 1614 Harney Street}

Omaha, Neb., January 10, 1920.

I have in stock the following Seeds, which I offer to the trade subject to prior sale and confirmation. Terms 60 days net, or $11 / 2 \%$ cash in ten days from date of invoice. Bags at value.

All seeds offered were grown either by myself or for me on a direct contract with the actual seed grower, and from very cafefully selected stocks, and are suitable for your most critical trade. Sam ples on application.

I have also good stocks of other seeds at reasonable prices. See regular wholesale list for prices on same.

Ask for prices and samples of alfalfa seed, Nebraska grown is the best.

Soliciting your valued orders, I remain.

Yours truly,
WERTER DE VAUGHN.

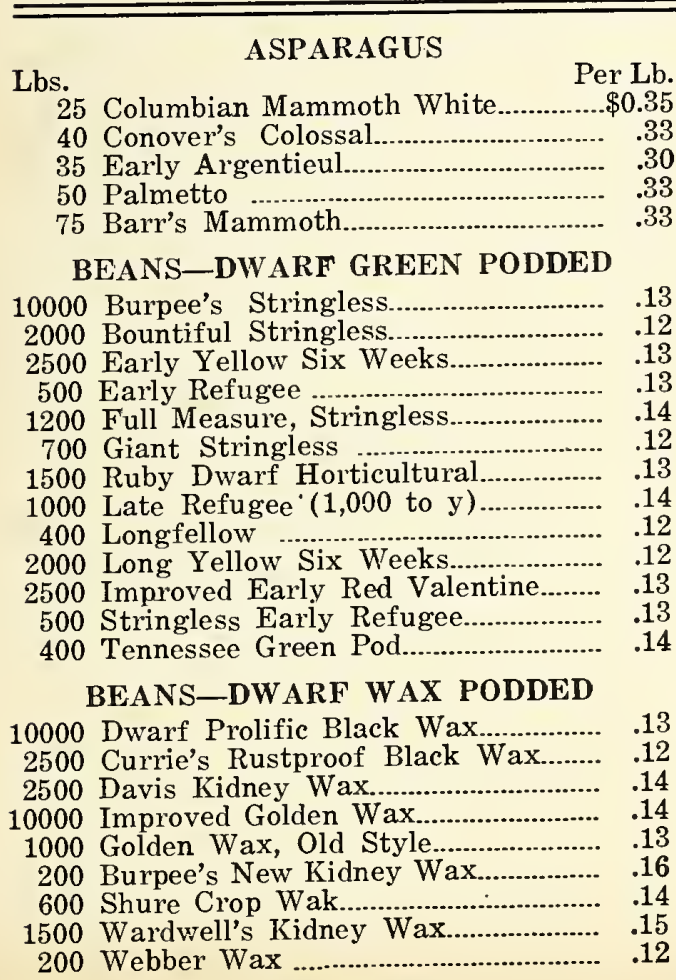

POLE LIMAS
$\$ 0.35$ .33

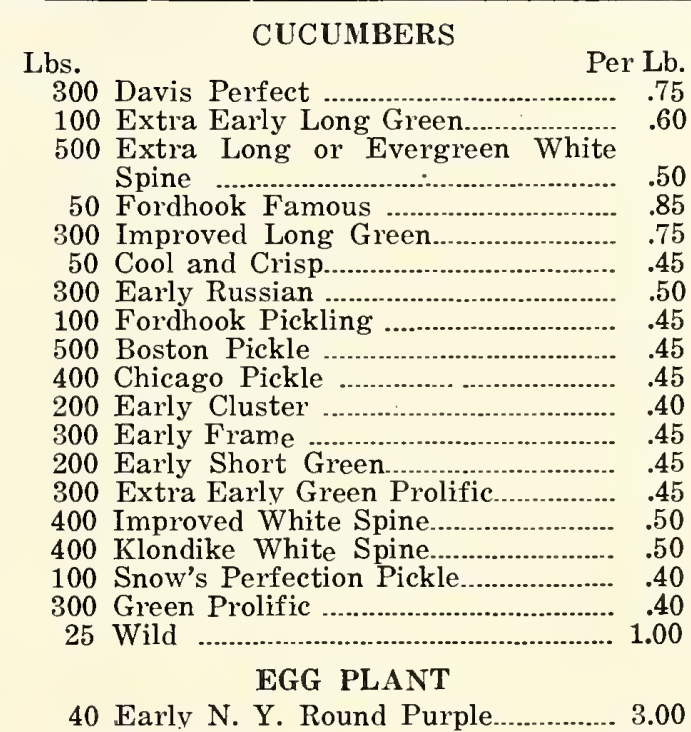

60 Black Beauty

\section{ENDIVE}

200 Broad Leaved Batavian..................... .40 200 Green Curled 100 Giant Fringed or Oyster...................... 200 Large Curled Pancalier.......................... .40 150 Large Green Curled............................. . .40 200 Moss Curled ......................................... .40
PEPPERS

Lbs.

15 Neapolitan

25 Ohio Crimson .................................... 5.00

35 Pimento ............................................... 2.50

25 Ruby King ....................................... 3.0

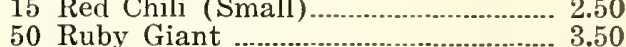

50 Ruby Giant ........................................... 3.50

5 Sweet Mountain ....................................... 3.00

50 Sweet Spanish ................................. 3.00

50 World beater ............

75 Giant Crimson .............. 4.50

PUMPKIN

250 Connecticut Field

25 Golden Cushaw (Small) 25 Japanese Pie

Kentucky Field

200 Quaker Pie .......................................... .85

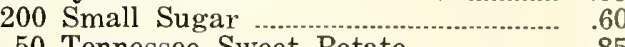

50 Tennessee Sweet Potato.................... .85

\section{RADISH}

Early Turnip and Globe Shaped

500 Crimson Giant Globe $\quad .50$

500 Early Turnip, Red or Scarlet_....... .35

2000 Early Turnip, Red, White Tip.......... .30

500 Early Scarlet Globe........................... .40

700 Perfection Round Red, White Tip Imported

2000 Rosy Gem

600 Sparkler Round Red, White Tip,

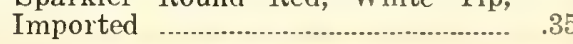



I have in stock the following Seeds, which $I$ offer to the trade subject to prior sale and confirmation. Terms 60 days net, or $1 / 2 \%$ cash in tell days from date of invoice. Bags at value.
All seeds of fered were grown either by myself or for me on a direct contract with the actual seed grower, and from very eafefully elected stocks, and are suitable for your most critical trade. Sam ples on application.

I have also good stocks of other seeds at reasonable prices. See regular
Ask for prices and samples of alfalfa seed, Nebraska grown is the best.

Ask for prices and samples of alfalfa seed, Nebraska grown is the best.
Soliciting your valued order's, I remain.
Yours truly,

WERTER DE VAUGHN

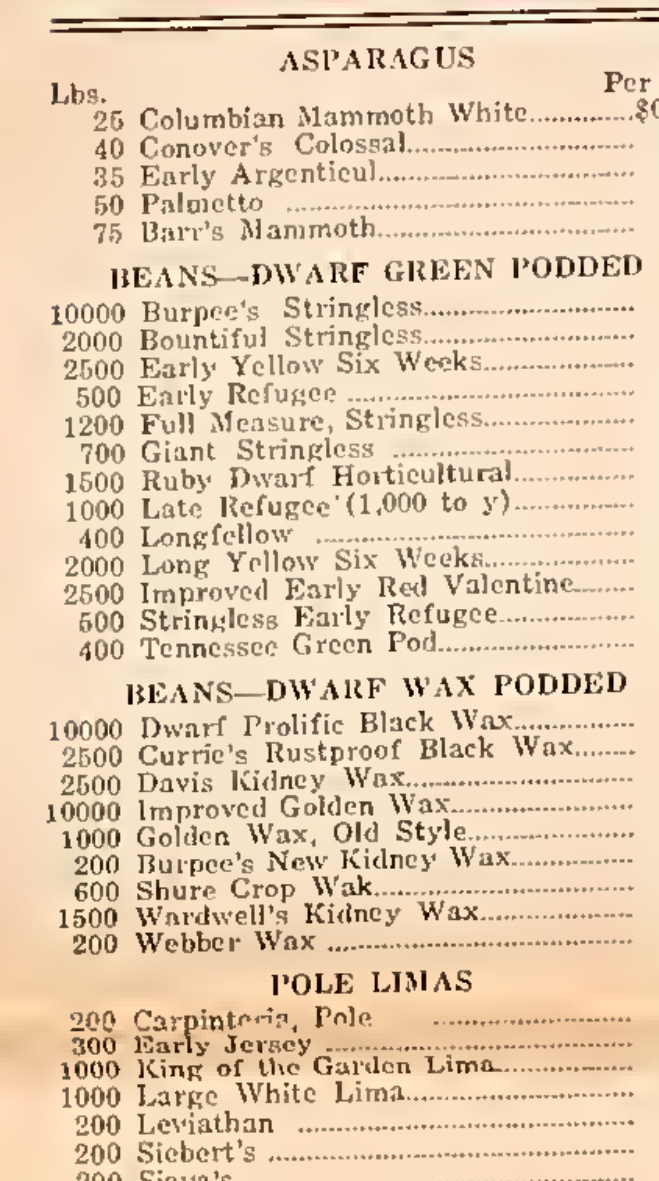

POLE BEANS-WAX PODDED

200 Golklen Cluster Wax....
700 Kientucky Wonder Wa

POLE BEANS GREEN PODDED

300 Saparagus Yard Long

100 Crease Back (Whitc)..............

100 Cut Shorts (Redl $\mathrm{Sp}$

BETTS FOR TABLE

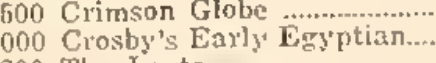

1000 Eimond's Blooil Turrip.

500 Detroit Dank Red.............

SWISS CILARD

HELT'S FOR STOCK

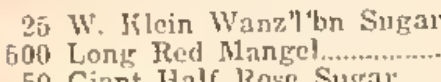

50 Giant Half Rose Sugar..........

600 Golden 'Tanka
100 Slud Strup Y'

BORECOLE OR KALE

BROCCOLI

30 Large White Leamington,... HRUSSELS SIROUTS

CABBAGE Early Varieties

50 Copenhagen Mlarket (Danisili..

Second Eariy Varictieg
75 Allhnost Early

75 Allhworl Early
110 Fairy Dwar' Fitat Dutch......
10 Henterson's Early Sunmer

Late V'arieties

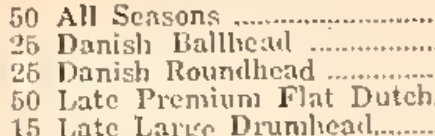

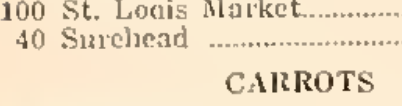

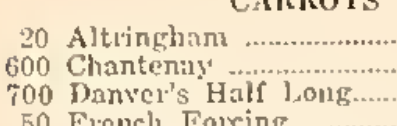

50 Frencll Forcing -.........

200 Long Orange ....................
300 Nante's (True French Strain).

500 Oxheart
300 Scallet Hom
150 White Bolgin

CELERY

20 Boston Market ............
40 Columbial
70 Giant Grolten Heart

f Blanching

COLLARDS

CRUSS OR PEPPER GRASS

SWEET COls
Farly Varietic

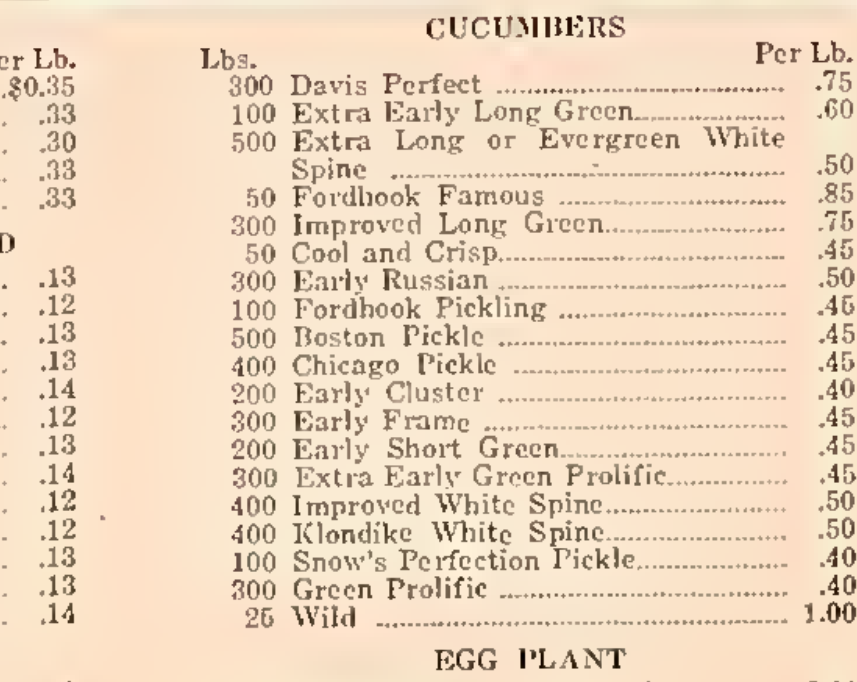

EGG PLANT
40 Farily N. Y. Round Purple 3.00
3.25

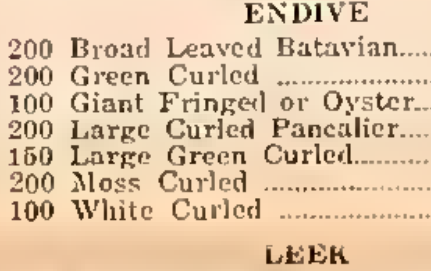

100 Amerícan Ligk FeK
50 Giant Mlusselburgh LETTUCE

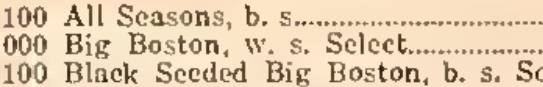

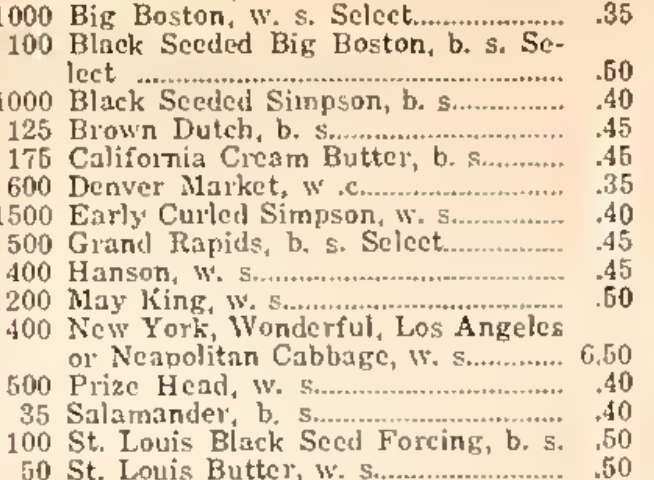

100 St. Louis Black Secd Forcing, b. s. 50 MUSKMELON

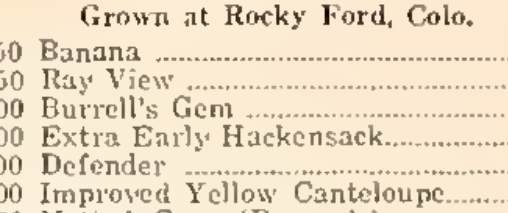

150 Netted Gem (Burpec's)....

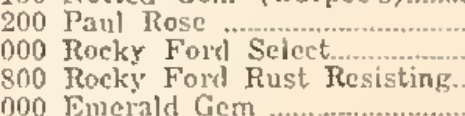

200 Millert's Crean

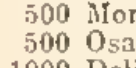
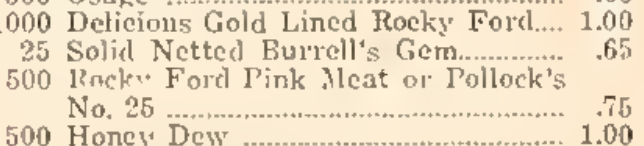

CASABA
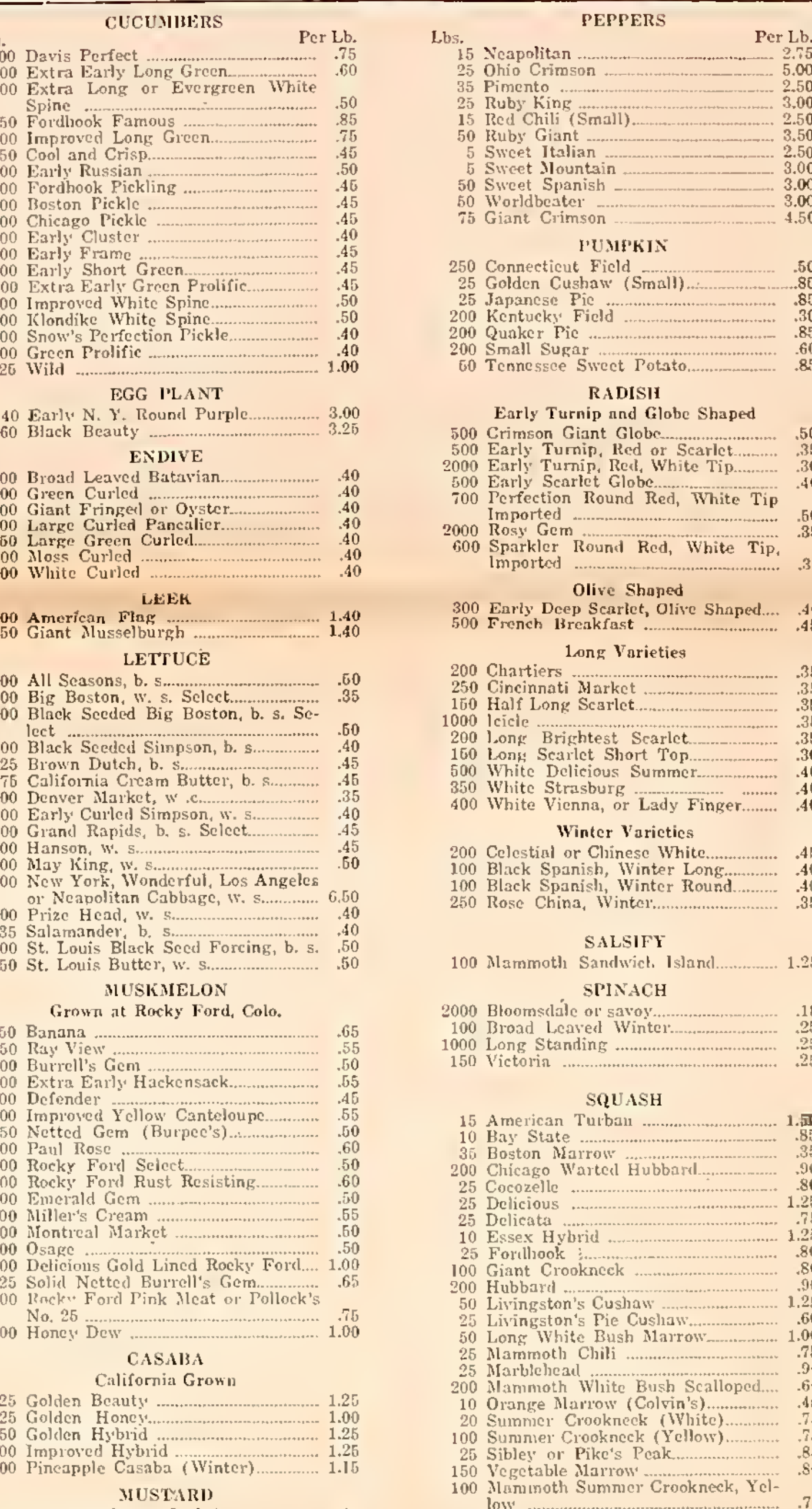

IUMPKIN

250 Connecticut Ficld
25 Golden Cushaw' (Smili)

200 Kentucks Fic

200 Small Sugar 60 Ternessee Swect Potato.

RADISII

Early Turnip and Globe Shaped

500 Crimson Giant Globe

500 Early Turnip, Red or Scarlet..........

500 Early Scarlet Globe.

600 Rparkler Round Red, White Tip.

Olive Shaped
300 Early Deep Scarlet, Olive Shaped....
500 French 13reakfast

1 ong Varieties

200 Chartiers .............
250 Cincinnati Market
160 Half Lon Scarlet.

200 loicie Brightest Scarlet

160 Lons Searlet Short Top

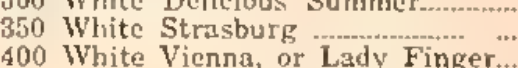
Winter Varictics

200 Celestini or Chinese Whitc...........
100 Black Spanish, Winter Long.

100 Black Spanish, Winter Round

SALSIFY SPINACH

2000 Btoomslale or savoy....

1000 Long Standin

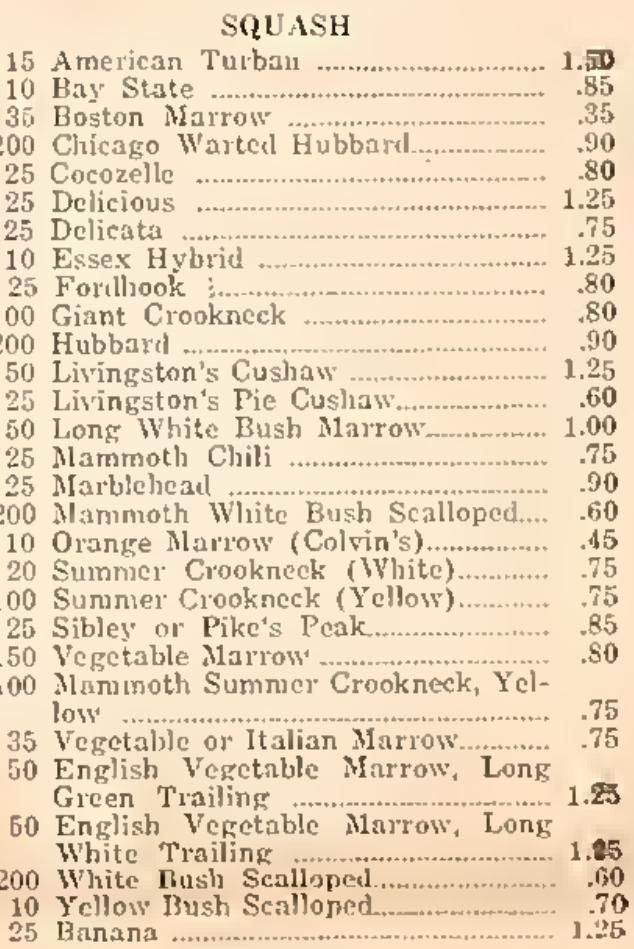

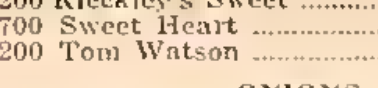

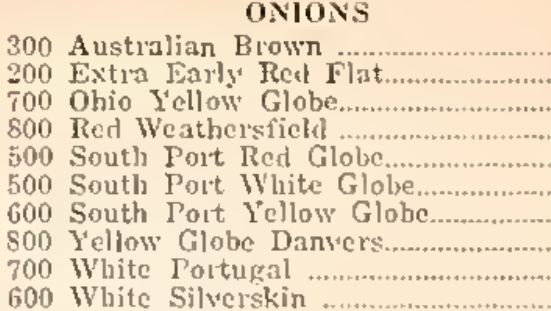

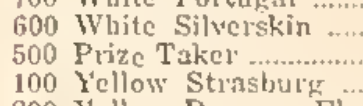

PARSLEY

100 Double Curlerl ............
200 Extra Triple Curled...
300 Moss or Triple Curled

50 Tumip Rooted ..........
75 Pinin or Single......

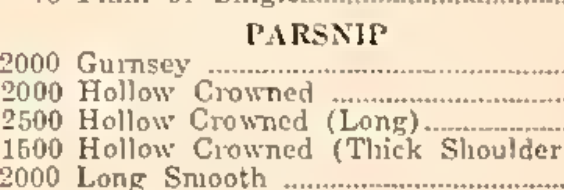

WRINKLED PEAS

1000 American
1000 Litle Mlarto
500 Lartonian

TONATO

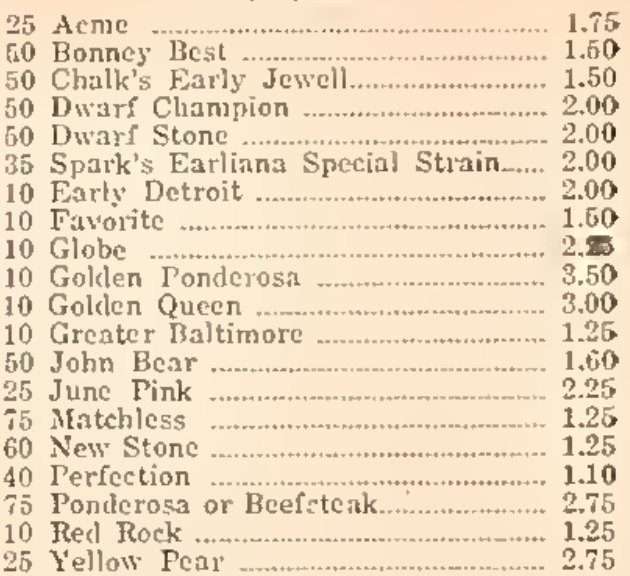

TURNIP

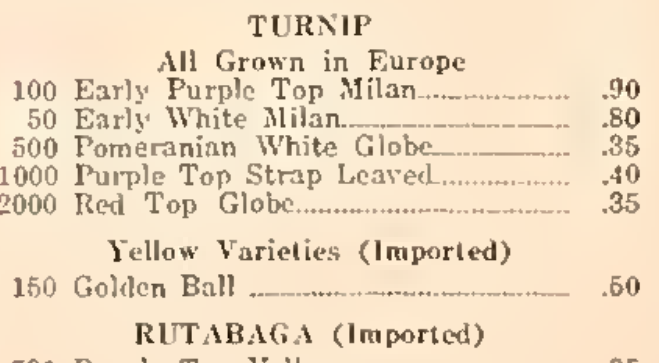

SWEET PEAS

500 Alaska

1000 White Eye Mlarrowf

1300 13lack
200
$2 u p$

1200 Best Large Flowering, Nixed...... 
